\title{
Physics and Evolution of LPVs from HIPPARCOS Kinematics
}

\author{
M.O. Mennessier and R. Alvarez ${ }^{1}$ \\ GRAAL, CNRS and Université MontpellierII, F-34095 Montpellier \\ Cedex 05, France \\ X. Luri \\ Departament d'Astronomia $i$ Meteorologia, Universitat de Barcelona, \\ E-08028 Barcelona, Spain \\ M. Noirhomme-Fraiture and E. Rouard \\ Institut d'Informatique, Université de Namur, B-5000 Namur, Belgium
}

\begin{abstract}
The HIPPARCOS satellite provides astronomical data for about one thousand Long Period Variable stars (LPVs) from which kinematics properties and luminosity calibrations in several bandpasses are deduced using an appropriate method. Several results are deduced: a classification of the LPVs and its relation with the classification from the light curves, a calibration of luminosities inducing properties (age, mass, etc.) along the AGB and a comparison of oxygen to carbon-rich stars.
\end{abstract}

\section{Introduction}

It is known that the evolution of LPVs strongly depends on their initial mass and metallicity. However a lot of questions remain open as, for instance, the evolution from $\mathrm{O}$ to $\mathrm{C}$-rich stars, the faintest and brightest luminosities on the AGB (see the reviews by Lattanzio \& Forestini, Blöcker, Mowlavi in this volume). Kinematical and photometric data allow to calibrate luminosities together with galactic properties. Using HIPPARCOS data we set out such results and their contribution to evolution problems.

\section{Luminosity calibrations and mean kinematical parameters}

\subsection{Samples and analyzing method}

The sample is composed of all LPVs observed by HIPPARCOS, i.e. the LPVs which are brighter than $\mathrm{V}=12.5 \mathrm{mag}$ during more than $80 \%$ of the time (around 900 stars). They are Miras, Semi-Regular or irregular L variables, O-rich, C-rich

\footnotetext{
${ }^{1}$ Now at Institut d'Astronomie et d'Astrophysique, Université Libre de Bruxelles, B-1050 Bruxelles, Belgium
} 
and S stars.

This sample is analyzed using the LM method of calibration (Luri, Mennessier et al. 1996) that needs apparent magnitudes and proper motions, and also uses available parallaxes (even not accurate ones) and radial velocities, and was implemented to:

- allow the treatment of non-homogeneous samples and determine the number of significant groups,

- take into account the effects due to sampling, interstellar absorption, observational errors,

- produce unbiased estimates of mean velocies (U, V, W), of axes of the velocity ellipsoid of each group $\left(\sigma_{U}, \sigma_{V}, \sigma_{W}\right)$ and of their mean scale height (z), and calibrate the mean luminosity and its dispersion $\left(\mathrm{M}, \sigma_{M}\right)$ of each group,

- calculate the a posteriori probability of a star to belong to a group and classify the stars into the groups, and estimate individual distances and luminosities.

Several calibrations were done, using successively:

- V magnitudes (882 stars) (results in HIPPARCOS Venice'97 Symposium)

- K magnitudes (652 stars)

- IRAS 12 and 25 magnitudes (793 stars) defined as:

$12=3.63-2.5 \log \left(F_{12}\right)$ and $25=2.07-2.5 \log \left(F_{25}\right)$

Let us note that 608 stars belong to both $\mathrm{K}$ and IRAS samples.

\subsection{Results of the calibrations}

A statistical Wilk's test allows to determine the significant number of groups. We find: 6 groups for the $\mathrm{V}$ analysis, 3 for the $\mathrm{K}$ analysis and 4 for the two IRAS analyses. Crossing $\mathrm{K}$ and IRAS gives 7 non-empty groups. The groups differ from one another by kinematics (i.e. ages and main sequence masses) and/or luminosities.

Fig. 1 shows the estimated distributions of parameters for each group from the $\mathrm{K}$ analysis. Each branch of the diagram corresponds to one parameter: $\left(\mathrm{U}, \sigma_{U}\right),\left(\mathrm{V}, \sigma_{V}\right),\left(\mathrm{W}, \sigma_{W}\right), \mathrm{z},\left(\mathrm{M}, \sigma_{M}\right)$. The axes of the velocity ellipsoid and the scale height are increasing from the diagrams $\mathrm{D}$ to TD and from the latter to ED. This can be interpreted as reflecting the disc (D), thick disc (TD) and extended disc (ED) galactic populations.

Fig. 2 shows a similar representation from the IRAS-12 analysis. The distributions of velocities and $z$ are similar as from the IRAS- 25 analysis. An increasing of the velocity ellipsoid and of the scale height is seen from the diagrams $\mathrm{D}$ to $\mathrm{TDb}$ and to ED with a similar interpretation as for the $\mathrm{K}$ analysis. However there is a fourth significant group (TDf) with kinematical and spatial distributions very similar to these of TDb but with different luminosity calibration: TDf is, in average, fainter (M12=-6.4 mag, M25=-6.5 mag) and TDb is brighter (M12=-7.9 mag, M25 $=-8.5 \mathrm{mag})$. So the analysis separates them because of envelope properties. Moreover D, TDb and ED have, in average, similar IRAS color indices: $12-25=0.6 \mathrm{mag}$ while TDf has a mean index of $0.1 \mathrm{mag}$ that points to a probable majority of stars with thin envelopes. 

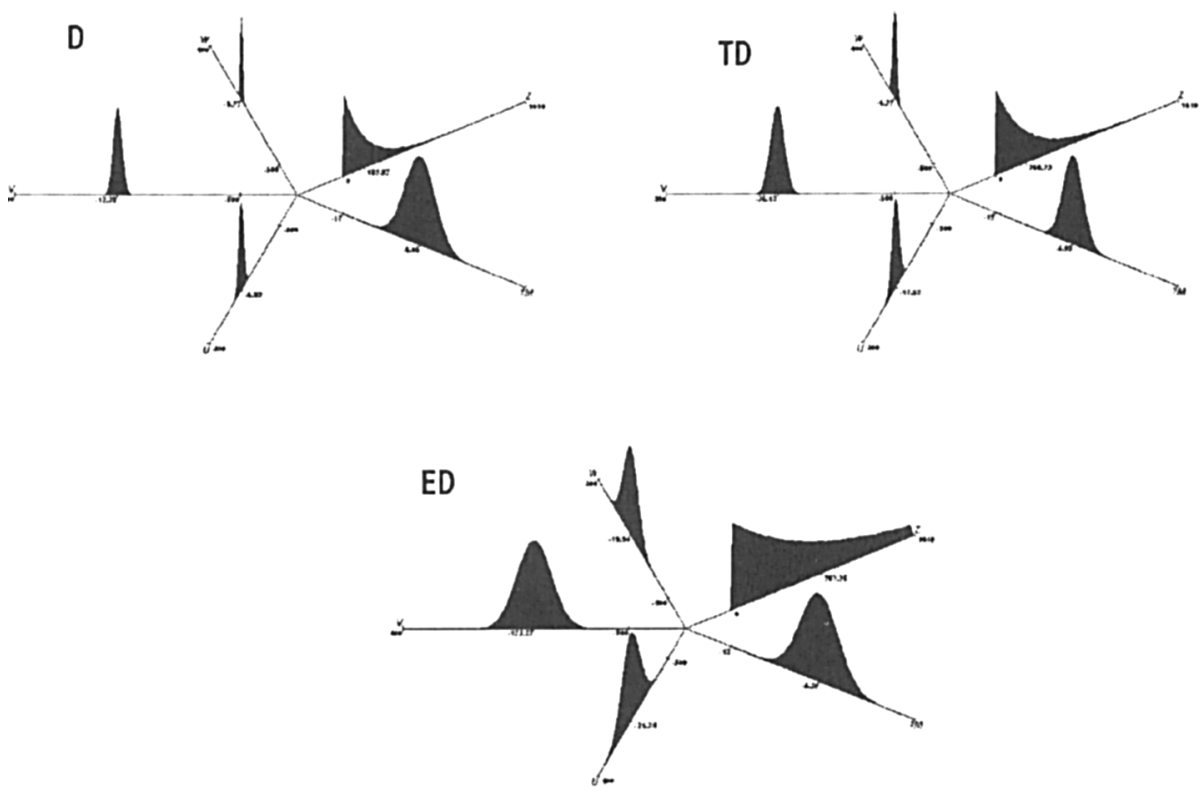

Figure 1. Distributions of estimated parameters from the K calibration
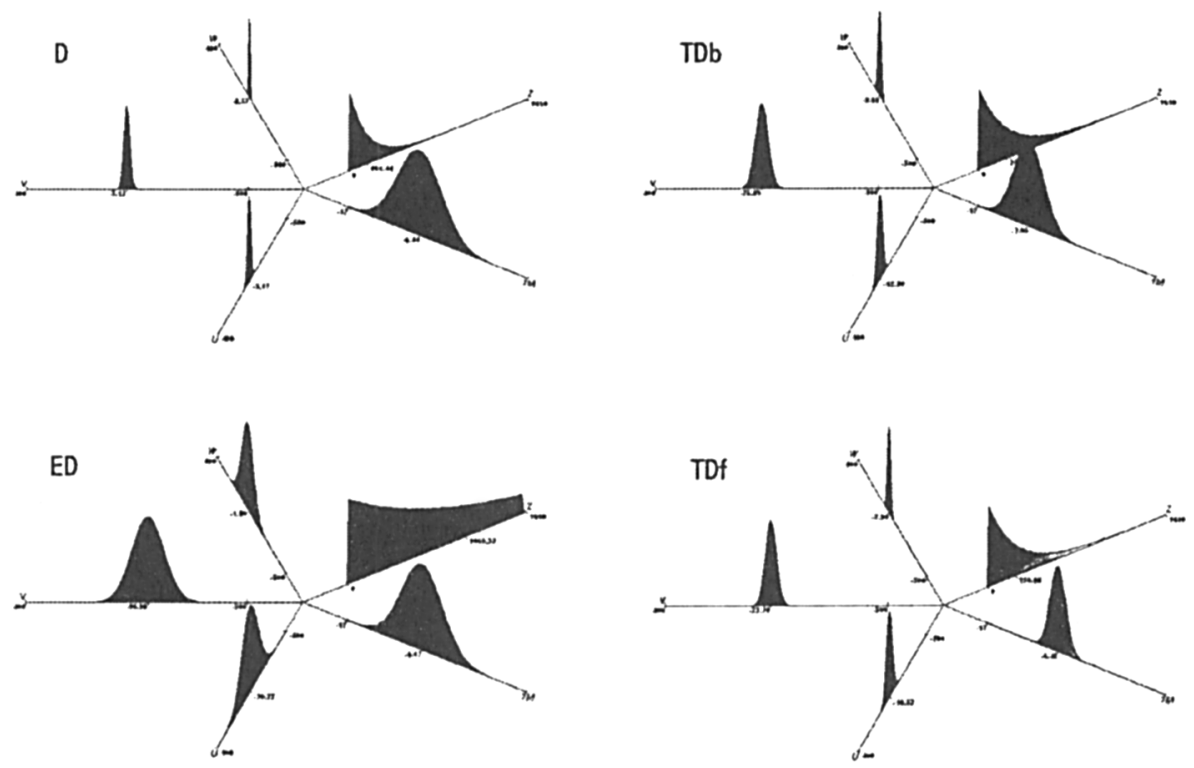

Figure 2. Distributions of estimated parameters from the IRAS calibration 


\section{Populations and variability types}

\subsection{Assignation of stars to groups}

For each analysis we a posteriori assign stars to one of the groups. In the following we focus on the classification when $\mathrm{K}$ and IRAS analyses are crossed: this classification results from both stellar parameters and envelope properties.

Fig. 3 shows the correspondence analysis of variability types and of crossed K-IRAS groups characterized not by the classical contingency table but by the attraction-repulsion indices. Each graph corresponds to one group denoted G $\left(G^{\prime}\right)$ where $G$ is the group from K-analysis and $G^{\prime}$ is the group from IRASanalysis. On each graph, each radius corresponds to one variability type (in clockwise): S-type SRb's and SRa's, C-rich, O-rich and S-type L variables, Crich, O-rich and S-type Miras, C-rich SRb's and SRa's, O-rich SRb's and SRa's. On each radius the attraction-repulsion index is indicated. The index is larger or smaller than 1 for attractive and repulsive groups respectively. A faint polygon at the value 1 and strong dots for the significant correlations are drawn.

By increasing ages and decreasing main sequence masses, the galactic groups are mainly correlated with:

- C-rich and S-type SR's and L variables [D(D)]

- C-rich variables and - but in a less proportion- O-rich Miras [TD(D)]

- O-rich SR's and Miras and C-rich Miras [D(TDb)]

- O-rich SRa's and Miras [TD(TDb) and ED(ED)]

Finally the groups $\mathrm{D}(\mathrm{TDf})$ and $\mathrm{TD}(\mathrm{TDf})$ are related to O-rich SRb's and irregular $\mathrm{L}$ variables.

This confirms that:

- nearly no C-rich stars are found in the groups corresponding to the lowest main sequence masses: the dredge-up may be more efficient for more massive stars (Busso et al. 1995) variables.

- SRa's seem close to the Miras while SRb's have a behaviour similar to $\mathrm{L}$

\subsection{Estimates of individual luminosities}

Individual allocation of one star to one group allows to estimate its most probable distance and so its most probable luminosity. Fig. 4 shows a luminosity-color diagram in function of the galactic groups:

- stars belonging to the disk population (i.e. with the highest main sequence mass) seem to go up higher on the AGB than stars belonging to the thick disk population,

- stars identified as belonging to the extended disk population go up along the AGB as high as disk population LPVs: the metallicity effect makes them brighter. 

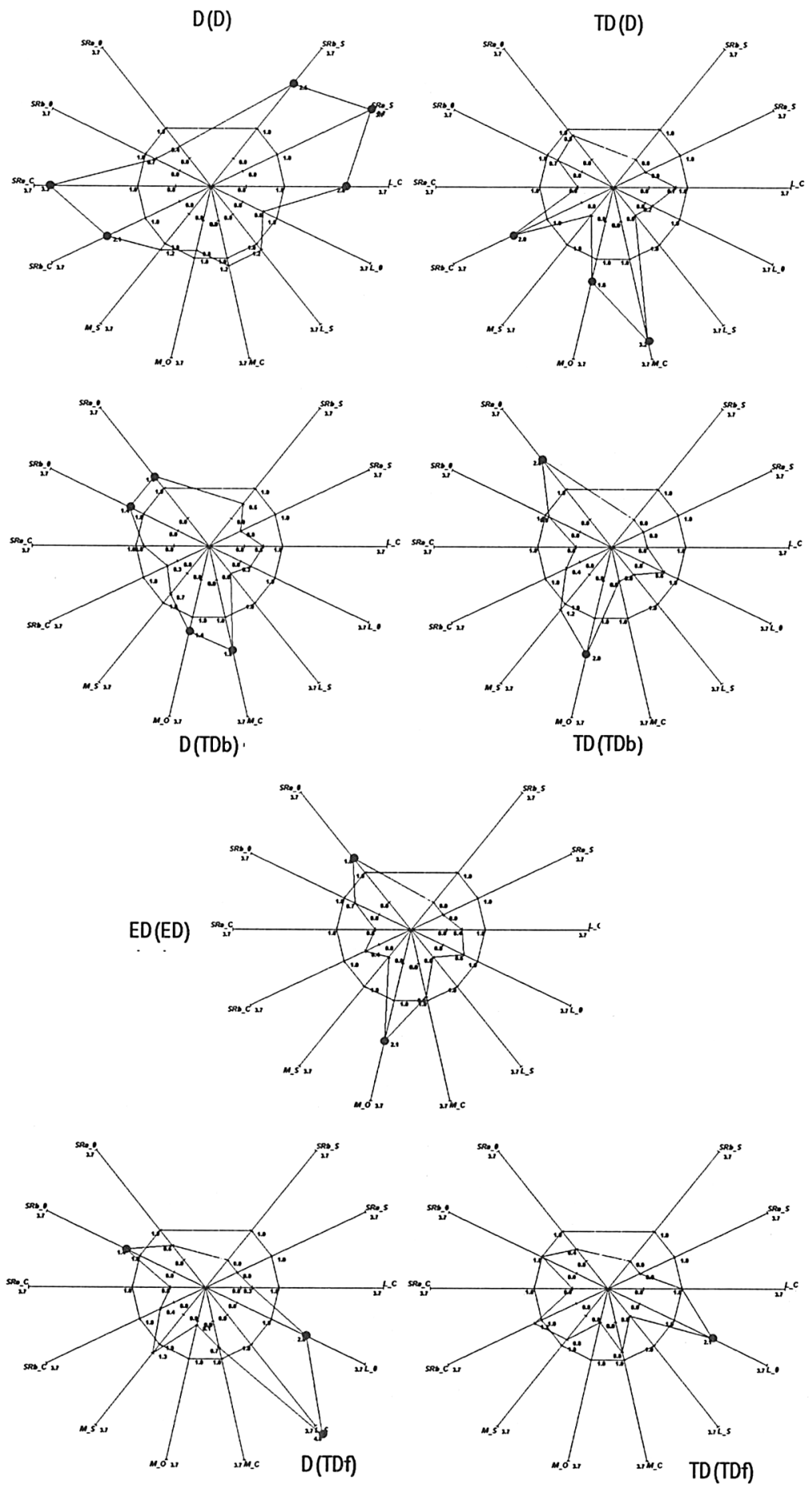

Figure 3. Correlations of variability types and K(IRAS) groups 


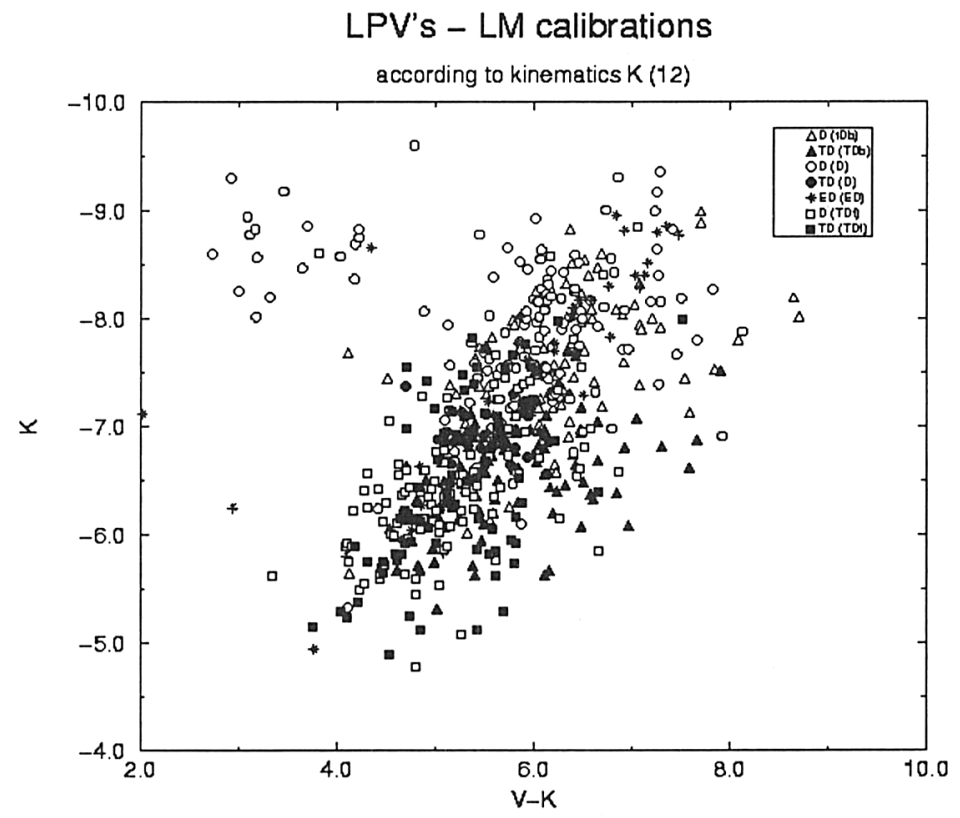

Figure 4. Color-luminosity diagram according to K(IRAS) groups

\section{Conclusion}

These first rough results that need to be refined show how much such considerations about luminosities joined to galactic properties could be a tool to constrain the models of evolution along the AGB.

But we must be very cautious with the conclusions because of possible biases due to a sampling effect: HIPPARCOS LPVs are the ones with the brightest optical counterparts. It will be necessary to include fainter LPVs which are bright infrared stars and LPVs without optical counterparts such as OH/IR stars. So it seems important to have an astrometrical and kinematical picture of the infrared sky in complement to photometric picture given by the IR surveys in course. The GAIA project is promising in this respect.

\section{References}

Busso M., Lambert D.L., Beglio L., et al., 1995, ApJ 446, 775

Luri X., Mennessier M.O., Torra J., Figueras F., 1996, A\&A 117, 405 